\title{
Advanced determination of piezoelectric properties of AlN thin films on silicon substrates
}

\author{
J.L. Sánchez-Rojas, J. Hernando \\ Dpto. Ingeniería Eléctrica, Electrónica, Automática y \\ Comunicaciones, E.T.S.I. \\ Industriales, Universidad de Castilla La Mancha, 13071 \\ Ciudad Real, Spain. \\ joseluis.sanchezrojas@uclm.es
}

\author{
A. Ababneh, U. Schmid \\ Saarland University, Faculty of Natural Sciences and \\ Technology II, Chair of \\ Micromechanics, Microfluidics/Microactuators, D-66123 \\ Saarbruecken, Germany.
}

\begin{abstract}
Piezoelectric deformations of thin, aluminum nitride (AIN) layers, on top of a silicon substrate, were studied by numerical calculations and interferometric measurements. Our calculation by finite element method demonstrates that substrate deformation under the top electrode may be comparable to the electric field induced deformation in the thin AIN layer, for a given applied voltage. Simulations also show the effect of a clamped or free substrate condition and the relative contributions of $d_{33}$ and $d_{31}$ piezoelectric constants. A Laser scanning vibrometry technique was used to measure deformations in the top surface with sub-picometer vertical resolution. By comparing calculations and experimental data, quantitative information about both $d_{33}$ and $d_{31}$ constants can be obtained.
\end{abstract}

Keywords-Piezoelectric constants; Aluminum Nitride; vibrometer

\section{INTRODUCTION}

Certain acoustic wave devices and micro-electromechanical systems (MEMS) use piezoelectric thin films as actuating elements. The fabrication of these devices on conventional silicon wafers is a common choice due to the possibility of integration with mature technologies [1-3]. Among the different piezoelectric materials, AlN is an interesting candidate for this integration process. Its good mechanical, chemical and dielectric properties, as well as the compatibility with CMOS technology, have determined its choice $[4,5]$. A number of complex techniques have been used to determine AlN piezoelectric properties, such as the piezoelectric coefficients $d_{33}$ and $d_{31}$ [6-11]. In this work, the piezoelectric performance of sputter-deposited AlN thin films on thick silicon substrate was simulated by FEM (Finite Element Model) analysis and characterized by scanning laser vibrometry. The simulation results show the displacement profile of the top and bottom surfaces of the thin film, as well as the displacement of the top and bottom surfaces of the substrate, for a free and a perfectly clamped structure. Besides, different characteristics of the displacement profiles were related to the transversal piezoelectric coefficient $d_{31}$. From an experimental point of view, the scanning laser vibrometer data supported the simulated results, with a good overall matching between the experimental and the calculated displacement profile of the top surface of the structure. In order to take advantage of the agreement between the experiment and the simulation along this profile, a fitting procedure was applied in order to evaluate the piezoelectric coefficients $d_{33}$ and $d_{31}$ accurately.

\section{STRUCTURES AND SIMULATIONS}

Figure 1 shows a schematic cross section of the metalpiezo-metal sandwich model for the simulation section. The thickness of the AlN layer and Si substrate is $2 \mu \mathrm{m}$ and 640 $\mu \mathrm{m}$, respectively. The material of the electrodes is Molybdenum (Mo) with a thickness of $0.2 \mu \mathrm{m}$ for both electrodes. For the experimental results, the main structure of the samples is similar to that of figure 1 .

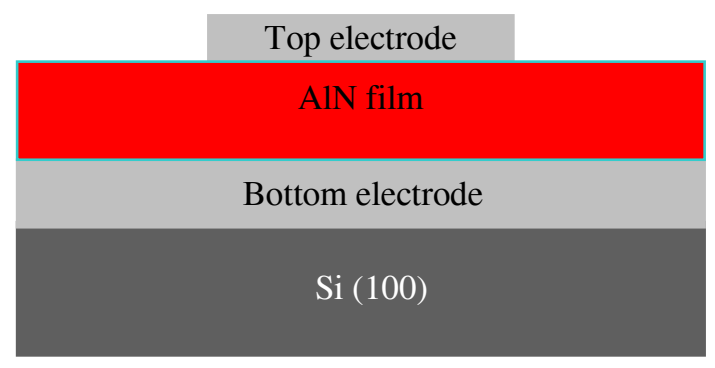

Figure 1. Device cross sectional view.

Next, we present the results of the FEM calculation for the structure of figure 1 with a perfectly clamped bottom face of the substrate. Figure 2 shows the results for a $200 \mu \mathrm{m}$ squareshaped electrode and an applied voltage of $17.6 \mathrm{~V}$. Figure $2 \mathrm{a}$ shows a cross sectional view perpendicular to the top electrode 
surface and along its centre. Only the $2 \mu \mathrm{m}$ thick piezoelectric layer and a part of the $640 \mu \mathrm{m}$ thick substrate are represented. Both electrodes are removed as they follow the deformation of the adjacent layers. The vertical displacement along the $\mathrm{Z}$ axis is magnified for a better view. The colour is also modulated following the total displacement. A quantitative representation is shown in figure $2 b$, where the $\mathrm{Z}$ displacement profiles of the top (solid) and the bottom (dashed) surfaces of the thin film are plotted against the $\mathrm{X}$ in-plane coordinate, whose origin is taken at the centre of the top electrode $(X=0)$. The origin of the vertical axis in this figure corresponds to the situation of no deformation for the curve under consideration.

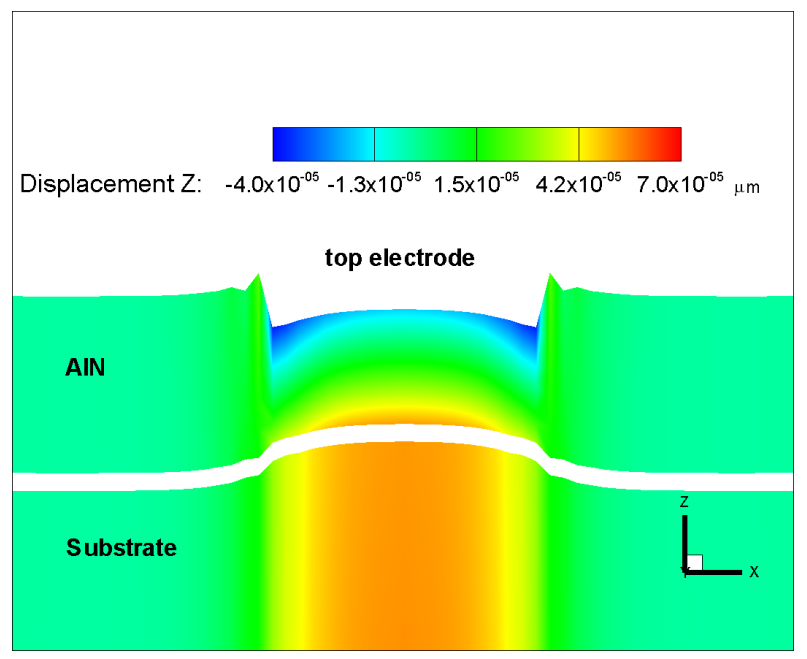

Figure 2a. FEM calculation of the deformation in AlN and silicon. Cross sectional view perpendicular to the top electrode surface. The vertical displacement along the $\mathrm{Z}$ axis is magnified for a better view

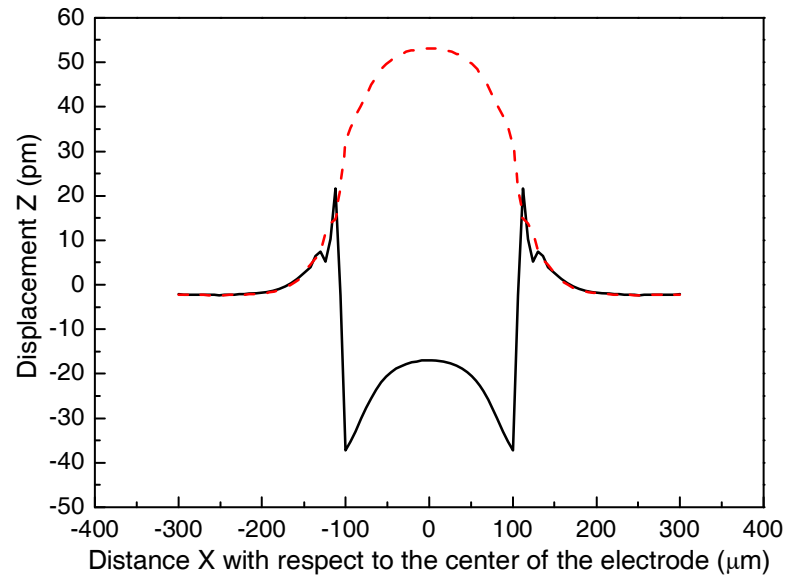

Figure $2 \mathrm{~b}$. $\mathrm{Z}$ displacement profiles (microns) of the top (solid curve) and the bottom (dashed curve) surfaces of the thin film against the $X$ in plane coordinate, whose origin is taken at the center of the top electrode $(X=0)$. The origin of the vertical axis corresponds to zero deformation.

The most important point to notice from this figure is the displacement of the bottom surface of the film. This displacement is far from being negligible, with a value of 53 $\mathrm{pm}$ at the centre of the electrode. For comparison, the displacement on the top surface is $-17 \mathrm{pm}$ at the same place. Therefore, for this electrode size, the total change of thickness in the piezoelectric layer is distributed at the top and the bottom surfaces. This fact brings out very serious limitations to the determination of the piezoelectric coefficient $d_{33}$ from a measurement of the displacement of the top surface of the structure, as it is the case with laser scanning interferometry and AFM [12.13]. In the way figure $2 b$ is presented, the electric field induced change of the film thickness at the centre of the electrode is directly given by the separation between the two curves at that point. Besides, the displacement of the bottom surface of the film is equivalent to the displacement of the top surface of the substrate. Consequently, there is a significant change in the substrate thickness below the top electrode. This would not occur at a perfectly rigid substrate. It is also worth mentioning that there is no bending of the substrate due to the boundary condition of perfect clamping.
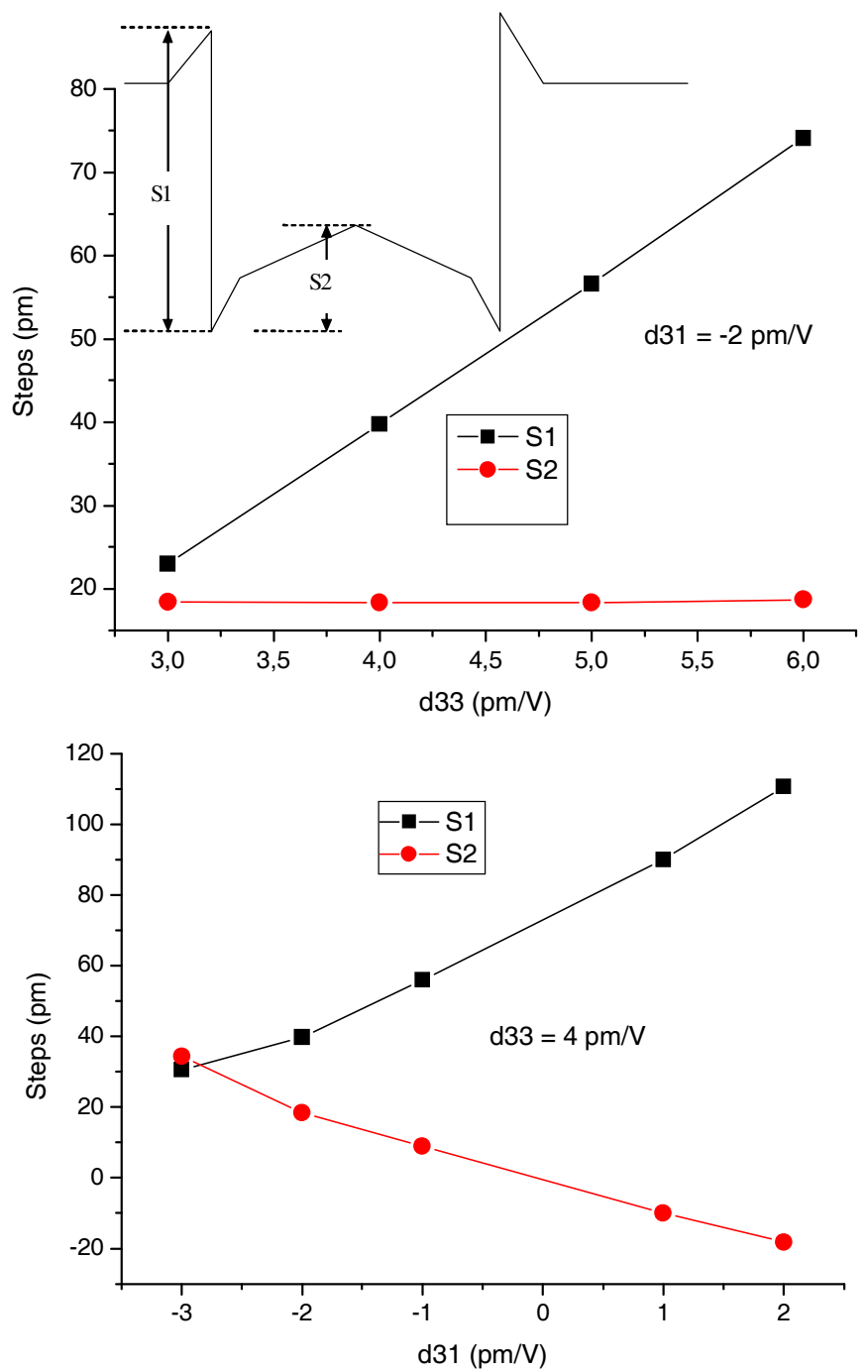

Figure 3. Magnitudes of the steps (as defined in the inset) at the edge (S1) and from edge to center (S2) against $\mathrm{d} 33$ and $\mathrm{d} 31$.

Figure 2 also shows the profile of the top surface of the film. This profile is characterized by different features around the edges of the top electrode. These features are the step in the 
displacement at the edges of the electrode (S1) and the nonuniform displacement at both sides of the step, characterized by the total displacement from edge to centre of the electrode and designated as S2 in the inset to figure 3 . The curvature of the top surface of the film below the top electrode is a result of the non-uniform displacement at both edges, below the top electrode.

Figure 3 demonstrates that $\mathrm{S} 2$ is mainly determined by $d_{31}$ and that is almost constant in a wide range of $d_{33}$ values close to those published in the literature. On the other hand, S1 is affected by both $d_{33}$ and $d_{31}$ with opposite trend, as expected. This behaviour will allow us to obtain both piezoelectric constants from an independent fitting of S1 and S2 to their experimentally obtained magnitudes.

\section{EXPERIMENTAL}

The experimental electric field-induced out-of-plane displacements were measured with a MSV 400 Polytec scanning laser Doppler vibrometer. The sample was placed on a probe station and electrical contacts to the top and bottom electrodes of the structure were made using tungsten dc tips. The system can scan a certain area of the device and more details are given in [12].

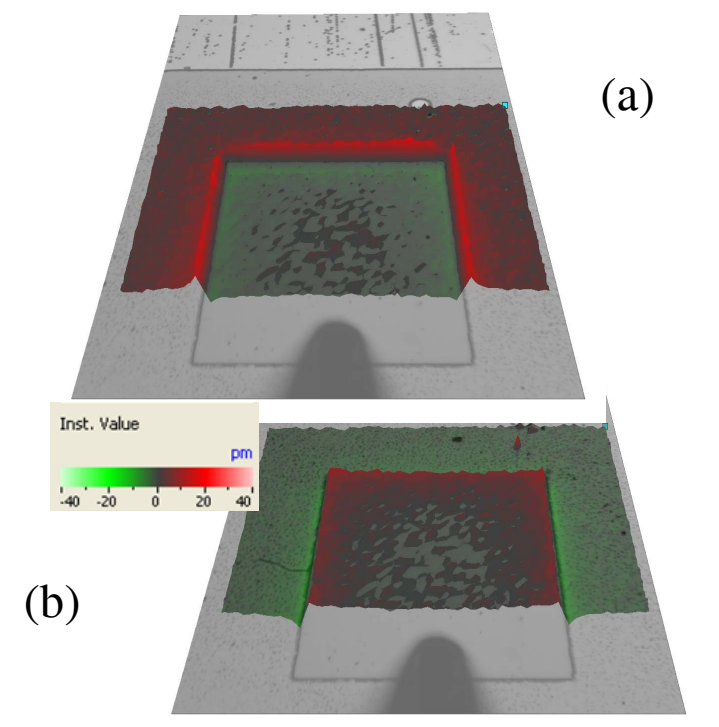

Figure 4. Scanning laser vibrometer measured displacement for a $2 \mu \mathrm{m} \mathrm{AlN}$ film on top of a $640 \mu \mathrm{m} \mathrm{Si} \mathrm{substrate.} \mathrm{The} \mathrm{area} \mathrm{shows} \mathrm{part} \mathrm{of} \mathrm{a} 200 \mu \mathrm{m}$ side square electrode and the surroundings, with the probe used to apply voltage to the structure.

For measuring the surface out of plane movements, the structure was excited by applying a sinusoidal signal between the electrodes with a given frequency. The amplitude of the applied sinusoidal signal was $17.6 \mathrm{~V}$ (nominally $18 \mathrm{~V}$, reduced by slight loading effects) in all the cases presented in this work. The frequency of the sinusoidal signal must be low enough and far enough from any mechanical resonance so that the experiment can be compared to the static model, for a given voltage amplitude. The scanning laser vibrometer was used to obtain an animation of the movement of a scanned area that included the top electrode and the surroundings for a structure glued to an $\mathrm{Al}$ holder in an attempt to clamp the bottom surface of the substrate, with a $2 \mu \mathrm{m}$ thick AlN film and a 200x200 $\mu \mathrm{m}^{2}$ square top electrode. Figure 4 shows the displacements obtained from the previous animation at (a) $17.6 \mathrm{~V}$ and (b) $-17.6 \mathrm{~V}$. The top electrode can be seen as well as the tip contacting it. The parameters of the measurements were given in the experimental procedure section. In this case, the frequency of the sinusoidal signal was $60 \mathrm{kHz}$, far from any mechanical resonance.

As the displacement profile measured experimentally resembles very well all the features of the simulated profile, it would be reasonable to obtain the piezoelectric coefficients by fitting the simulated profile to the experimental one. In this way, the whole profile, and not only a particular feature such as the step, would be used for the evaluation of the piezoelectric coefficients, although the results of figure 3 regarding steps S1 and $\mathrm{S} 2$ can be used as a guide. The measured profile must not be affected by any resonance of the structure in the tens of $\mathrm{kHz}$ range. According to our measurements this implies the use of top electrodes with a side of $200 \mu \mathrm{m}$ or smaller, as well as gluing the structure to a holder. The results obtained under these conditions can be compared to the simulations for a perfectly clamped structure.

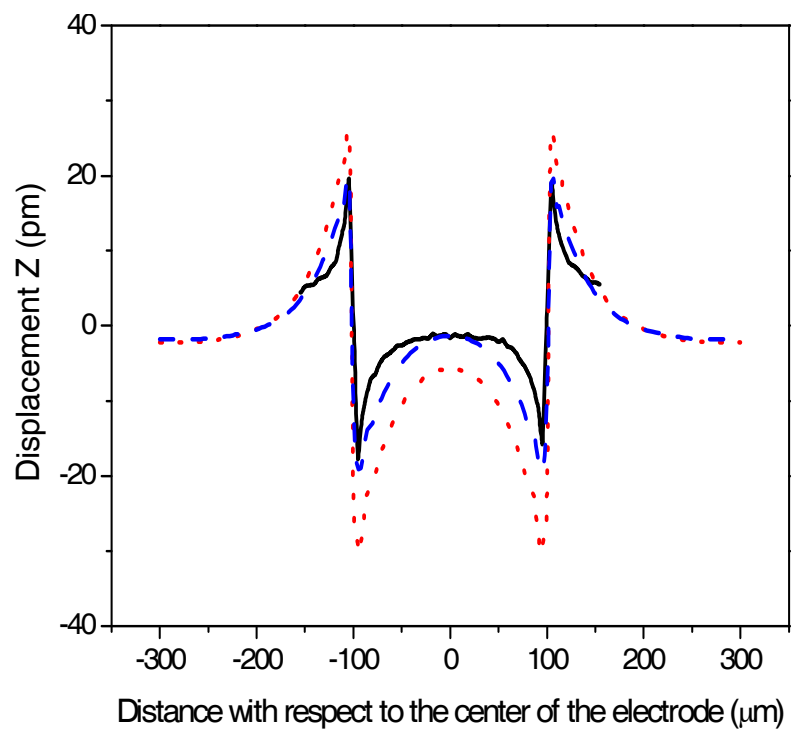

Figure 5. Fitting to the experimental result with the simulations. The solid line corresponds to the average of different measured displacement profiles along a $200 \mu \mathrm{m}$ side square top electrode for a $1.8 \mu \mathrm{m}$ AlN glued structure. The dotted line is the calculated result for $d_{33}=5.5 \mathrm{pm} / \mathrm{V}$ and $d_{31}=-2.6 \mathrm{pm} / \mathrm{V}$. The dashed line is the simulation for $d_{33}=4 \mathrm{pm} / \mathrm{V}$ and $d_{31}=-2 \mathrm{pm} / \mathrm{V}$.

Figure 5 shows a fitting example. The solid curve is an average of the measured profiles over a $200 \times 200 \mu \mathrm{m}^{2}$ square top electrode for a glued, $1.8 \mu \mathrm{m}$ thick, AlN structure. The sinusoidal excitation signal had $17.6 \mathrm{~V}$ peak at $60 \mathrm{kHz}$. This 
curve is characteristic of the many different measurements performed in different samples. Two calculated displacement profiles are also included in the figure, associated with two sets of piezoelectric coefficients. The dotted curve corresponds to values for $d_{\mathrm{ij}}$ obtained from previous works. In this case, the measured curve is less pronounced and has lower vertical displacements than predicted by the model. Next, we tried to find the values of $d_{33}$ and $d_{31}$ that best fit the experimental curve for this sample; In our case, we found a good agreement with the dashed curve, which corresponds to $d_{33}=4 \mathrm{pm} / \mathrm{V}$ and $d_{31}=-2 \mathrm{pm} / \mathrm{V}$. With these values, the step at the edges of the top electrode and the curvature under the top electrode are comparable for both the experiment and the simulation. In this way, the estimated piezoelectric coefficients are deduced from a comparison between experiment and theory that takes into account the whole displacement profile and not only a particular point or feature in the measurement.

\section{CONCLUSIONS}

AlN piezoelectric thin films were studied using FEM simulations and scanning laser vibrometry, in terms of the displacement generated by the voltage applied between the top and bottom electrodes, on top of a silicon substrate. Our calculations, taking into account the compliance of the substrate, predict a pronounced deformation of the bottom surface of the thin film located below the top electrode, and consequently of the top face of the substrate, for the top electrode sizes commonly used in the literature. Therefore, the thin film thickness variation is thus the result of both the top and bottom surface displacements. Since techniques such as scanning laser vibrometry measure only the displacement on the top surface, the change in film thickness can not be directly deduced. As a result, the immediate determination of the piezoelectric coefficient $d_{33}$ from the measurement of the displacement of the top surface of the structure is not straightforward. In our case, based on the excellent agreement between the experimental and the calculated displacement profiles, the values of $d_{33}$ and $d_{31}$ in AIN were deduced by applying a fitting procedure that includes the different features of the profile along the top electrode.

\section{ACKNOWLEDGEMENTS}

This work has been supported by Junta de Comunidades de Castilla-La Mancha under project number PCC08-0015-0722 and by Spanish Ministerio de Educación y Ciencia under project number DPI2006-01923.

\section{REFERENCES}

[1] B. Piekarski, D. DeVoe, M. Dubey, R. Kaul, J. Conrad, Sensors and Actuators A, 91 (2001), pp 313-320.

[2] G. Perçin, B.T. Khuri-Yakub, Ultrasonics 40 (2002), pp 441-448.

[3] P. Cong, T-L. Ren, L-T. Liu, Microelectronic Engineering, 66 (2993), pp 779-784.

[4] E. Iborra, M. Clement, J. Sangrador, A. Sanz-Hervás, L. Vergara, M. Aguilar, IEEE Transactions on Ultrasonics Ferroelectrics, and Frequency Control, 51 (2004), pp 352-358.
[5] Günther, M. Egretzberger, A. Kugi, K. Kapser, B. Hartmann, U. Scmid, H. Seidel, IEEE Sensors Journal, 6 (2006), pp 596-604.

[6] E. Iborra, L. Vergara, J. Sangrador, M. Clement, A. Sanz-Hervás, J. Olivares, IEEE Transactions on Ultrasonics, Ferroelectrics, and Frequency Control 54 (2007), pp 2367-2375.

[7] M-A. Dubois and P. Muralt, Applied Physics Letters, 74 (1999), pp 3032-3034.

[8] J. A. Christman, R.R. Woolcott, Jr., A.I. Kingon, R.J. Nemanich, Applied Physics Letters, 73 (1998), pp 3851-3853.

[9] A. L. Kholkin, Ch. Wütchrich, D. V. Taylor, and N. Setter, Review of Scientific Instruments, 67 (1996), pp. 1935-1941.

[10] M-A. Dubois and P. Muralt, Journal of Applied Physics, 89 (2001), pp 6389-6395.

[11] R. Herdier, D. Jenkins, E. Dogheche, D. Rèmiens, and M. Sulc, Review of Scientific Instruments, 77 (2006), pp 093905.

[12] Hernando, J.; Sánchez-Rojas, J. L.; González-Castilla, S.; Iborra, E.; Ababneh, A.; Schmid, U.. Journal of Applied Physics, Sep2008, Vol. 104 Issue 5, pN.PAG, 9p; DOI: 10.1063/1.2957081; (AN 34360647)

[13] Z. Wang and J. Miao, J. Phys. D 41, $035306 \_2008$. 